\title{
A Modified Matrix Algorithm Dichotomy for Distribution Network Fault Location
}

\author{
Hanyu Yan ${ }^{1,}{ }^{*}$, Xueying $\mathrm{Wu}^{2}$, Chunlan $\mathrm{Lin}^{2}$, and Haiyan $\mathrm{Liu}^{2}$ \\ ${ }^{1}$ Guangxi Power Grid Co. ,Ltd. Liuzhou Power Supply Bureau Liuzhou, Guangxi,China \\ ${ }^{2}$ Guangxi University of Science\& Technology School of Vocational \& Technical Education Liuzhou, \\ Guangxi, China
}

\begin{abstract}
The operation mode of distribution network is diversified, and it is difficult to accurately locate the fault point when the fault occurs in the case of multiple power sources and branches. In order to accurately locate the fault point and quickly restore the power supply in the fault-free area, a bisection fault location method based on improved matrix is proposed. According to the feedback information of terminal feeder equipment, the improved matrix algorithm is used to form the grid structure matrix and fault information matrix base on the connection relationship of distribution network. The grid structure matrix reflects the connection relationship between nodes and the power flow direction of the distribution network in normal operation. The fault information matrix is the flow direction of power flowing through terminal equipment when the system fails. According to the grid matrix and fault information matrix, the fault judgment matrix is obtained to judge the fault area. Finally, the dichotomy method is used to judge the fault area and determine the specific fault point. The effectiveness and practicability of the method are verified by an example.
\end{abstract}

Keywords: Distribution network; fault location; dichotomy; improved matrix algorithm

\section{Introduction}

With the rapid economic development, the load of the distribution network continues to increase, and its network branch connections are more complicated. In order to improve the reliability and stability of power supply in distribution networks, many distribution networks currently use multi-power or loop-circuit operation. In addition, considering the random and fluctuating renewable power and new energy vehicle access, when the line of the distribution network fails, it will bring certain difficulties to the precise location of the line fault. Therefore, it is greatly significance for improving the reliability and stability of the distribution network and shortening the power failure time, that finding a fault location method suitable for the distribution network under complex operating modes.

The traditional fault location methods of the radial distribution network often include

*Corresponding author: wuxueying830@163.com 
the impedance method, the traveling wave method, and the signal injection method. Among them, the impedance method is greatly affected by the system operation and short-circuit, and the accuracy of measuring the impedance is not high, so The distribution network is no longer used; although the traveling wave method is not affected by the type of fault and the length of the line, it has certain effectiveness for single-source radiating lines, but the complex structure of the multi-power distribution network has certain limitations; the signal injection method is for single-phase ground faults are feasible, while other types of faults are not accurate, and the injection method requires special equipment and a lot of investment. As the intelligence of automation equipment constantly improve, feeder terminal equipment, fault indicators and other related equipment are gradually used in the distribution network, and grid maintenance personnel make fault location judgments based on the fault information of the fault indicator or feeder terminal equipment. Therefore, the determination of the fault location relies heavily on the fault data information of the terminal equipment, such as the failure of the fault information or the false alarm indirectly increases the difficulty of fault finding. In [1], in order to reduce the false alarms and false alarms of feeder terminal equipment, it is proposed to establish a distribution network fault location analysis model to continuously reduce the dimension of the objective function variable, hierarchical analysis model, and continuous hypothesis testing to determine the fault location. Considering that the line location of line faults depends on the fault information transmitted by the feeder terminal equipment, the fault tolerance of smart equipment is poor. Therefore, the fault location problem is transformed into an integer programming problem, and a sine-cosine optimization algorithm is proposed [2], which is applied to the distribution network fault area location. This method is superior to fault location accuracy and rapidity when multi-point faults and fault information are missing Genetic algorithm and particle swarm algorithm. In [3], the concept of fault information correction is introduced to solve the problem of misjudgment of fault location due to fault information distortion of feeder terminals. In order to improve the accuracy of fault location in distribution network and reasonably use the automation equipment of distribution network, the matrix algorithm combined with feeder terminal equipment to transmit fault information and the graph theory considering the structure of distribution network was used to realize fault location. In order to generate the grid structure information and search the fault point in a certain positive direction, when facing the multi-power and large-scale grid, it is necessary to assume the positive direction to search respectively, which requires a large amount of calculation. Aiming at the problem that the traditional matrix algorithm's multipower source fault location has a large amount of calculation and the difficulty of multisource and T-junction area fault judgment, an improved matrix algorithm is proposed [4]. This method is based on the grid structure and Kirchhoff. The current law further improves the fault criterion in the T-node area, and can correctly determine the fault point when the FTU upload information is incomplete or distorted. In order to improve the tolerance and calculation speed of the matrix algorithm, combination of the optimization algorithm with the matrix algorithm is proposed [5]. In order to reduce the amount of calculation between matrices, combined with the characteristics of the distribution network structure, an improved matrix algorithm is proposed [6]. The logical operation between the matrices is used to replace the matrix multiplication or normalization operation. The calculation amount is greatly reduced, and it is also suitable for Multi-source and multi-point failure conditions. In addition, many research scholars have combined artificial intelligence algorithms with feeder terminal equipment to achieve fault location. In order to solve the problem of information distortion in FTU, the concept of fault information correction is introduced [7], and the particle swarm optimization algorithm is used to solve the model iteratively. In order to find the fault points effectively under the condition of multi-point fault and information distortion in distribution network, a two-state binary particle swarm 
optimization algorithm is proposed to construct the evaluation function of fault location, and the problem of local early convergence is considered [8]. According to the switching problem of distributed generation, an improved bacterial foraging algorithm based on differential evolution is proposed [9] for fault location of distribution network. Combined with the idea of region division, DG switching area is divided into active and passive trees to reduce the solution space. The global optimization ability of the algorithm is realized through the coordination of diversity control and cross operation. The fault finding of complex multi-source distribution network verifies its effectiveness efficiency and fault tolerance. The above-mentioned documents are aimed at the radiant distribution network or multi-source and each distribution network switch is equipped with intelligent fault judgment equipment such as feeder terminal equipment or fault indicator, and the actual feeder terminal equipment of the distribution network equipment, the quantity and accuracy are far inferior to the transmission grid. The distribution network has many load switches and low automation. The corresponding feeder terminal equipment is installed at important load nodes. Therefore, according to the feedback information of the terminal equipment, it can only know that a fault occurs in a certain area, and it is impossible to accurately determine the fault point. It is also necessary for the relevant maintenance personnel to patrol the line to find the fault point. If the feedback information is distorted or lost, the repair personnel will be found to find the fault.

To solve this problem, this paper proposes a dichotomy fault location method based on improved matrix. This method uses real-time terminal feeder equipment feedback information to form a grid structure matrix reaction distribution network connection relationship and power flow direction, fault information matrix reaction system after the fault, the flow of power flow is obtained based on the grid matrix and fault information matrix operation to obtain the fault judgment matrix for judging the fault area. Secondly, use the dichotomy method to judge the fault area to determine the specific fault point. Finally, the simulation results of numerical examples show that the method has significantly improved the accuracy of fault location and the speed of finding faults, shortened the time of power failure investigation, and improved the reliability and stability of the distribution network.

\section{Principle of improved matrix algorithm}

\subsection{Grid information matrix}

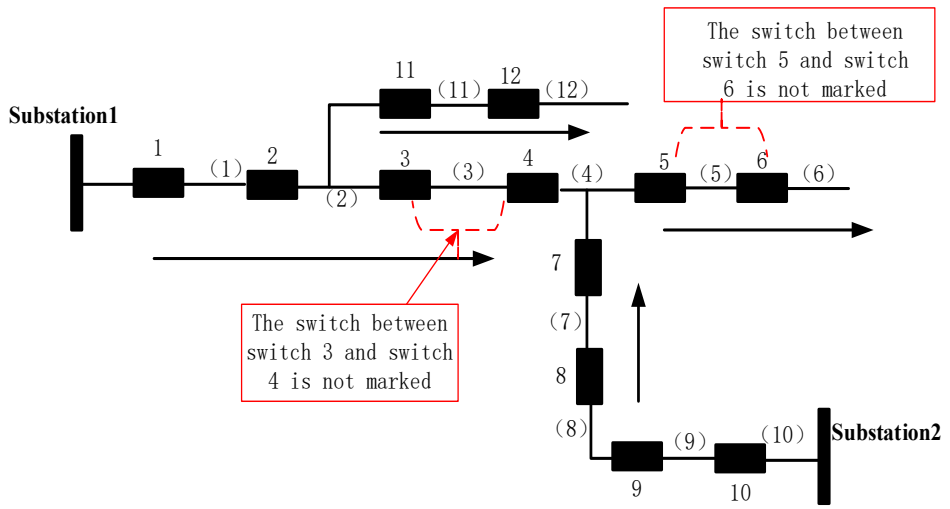

12:smart switch (1)-(12):line section

Figure 1. Grid with dual power supply mode 
A line in a substation is shown in figure 1. The grid in the figure shows the line connection relationship and label of the feeder terminal equipment installed on the line. The switch nodes without feeder terminal equipment are not marked on the grid, such as the switch on the line There are still many switches between 3 and switch 4 or between switch 5 and switch 6. Because no device is installed, the signal cannot be fed back, so it cannot be reflected in the information matrix. The black square in the figure is the node where the feeder terminal equipment has been installed, and the terminal equipment is the number of the line area between the nodes. For example, the section between node 1 and node 2 is the area (1), between node 5 and node 6 is area (5). Based on these connection relationships, the aforementioned grid matrix is generated. Each row of the matrix represents the connection relationship of an area, and each corresponding column represents a switch node. As shown in figure 1, node 2 is connected to area (1) and the positive flow direction of switch 2 points to area (1), then the matrix $Y_{12}=1$; if node 2 is connected to area (1) and positive flow direction of switch 2 deviates from the area (1), then the matrix $Y_{12}=-1$; if the node 4 is not directly connected to the area (1), then the matrix $Y_{14}=0$. Therefore, the generator matrix needs to define a positive direction, which plays an important role in the subsequent fault matrix judgment. How to stipulate the positive direction, if the power source position is the positive direction, when the operation mode changes, such as multipower supply, the positive direction at this time will be easily confused, and even need to reset the positive direction. In summary, when the distribution network is in normal operation, the direction of the power flow through the switch is the positive direction. When the operation mode changes, the element values of the matrix $Y$ are modified according to the feedback information ${ }^{[10-12]}$.

Assuming that the direction indicated by the black arrow in figure 1 is the power flow direction of the actual power grid operation, the positive direction of the switch is determined according to this flow direction. At this time, the grid matrix $Y$ of figure 1 can be determined according to the connection relationship between the switches and the flow direction.

$$
Y=\left[\begin{array}{cccccccccccc}
1 & -1 & 0 & 0 & 0 & 0 & 0 & 0 & 0 & 0 & 0 & 0 \\
0 & 1 & -1 & 0 & 0 & 0 & 0 & 0 & 0 & 0 & -1 & 0 \\
0 & 0 & 1 & -1 & 0 & 0 & 0 & 0 & 0 & 0 & 0 & 0 \\
0 & 0 & 0 & 1 & -1 & 0 & 1 & 0 & 0 & 0 & 0 & 0 \\
0 & 0 & 0 & 0 & 1 & -1 & 0 & 0 & 0 & 0 & 0 & 0 \\
0 & 0 & 0 & 0 & 0 & 1 & 0 & 0 & 0 & 0 & 0 & 0 \\
0 & 0 & 0 & 0 & 0 & 0 & -1 & 1 & 0 & 0 & 0 & 0 \\
0 & 0 & 0 & 0 & 0 & 0 & 0 & -1 & 1 & 0 & 0 & 0 \\
0 & 0 & 0 & 0 & 0 & 0 & 0 & 0 & -1 & 1 & 0 & 0 \\
0 & 0 & 0 & 0 & 0 & 0 & 0 & 0 & 0 & -1 & 0 & 0 \\
0 & 0 & 0 & 0 & 0 & 0 & 0 & 0 & 0 & 0 & 1 & -1 \\
0 & 0 & 0 & 0 & 0 & 0 & 0 & 0 & 0 & 0 & 0 & 1
\end{array}\right]
$$

It can be seen from the matrix $Y$ that the non-zero elements of each row of the matrix are the number of branches connected to the corresponding area, and each column represents a node. The sum of the values of each column of the matrix is equal to zero, which meets Kirchhoff's current law, and the current flowing into the node is equal to the current flowing out of the node. 


\subsection{Fault information matrix and fault judgment matrix}

When a fault occurs in a certain area of the distribution network in figure 1, the power flow in the distribution network changes as each power supply delivers power to the fault point. The feeder terminal equipment detects the change in the flow direction and magnitude of the current and feeds it back to the system. According to the above positive direction, the working mode of the feeder terminal equipment is set to $-1,1,0 . "-1$ " indicates that the fault current at the switch is opposite to the set positive direction; "0" indicates that no fault current is detected; "1" indicates that the fault current at the switch is the same as the set positive direction ${ }^{[4]}$.

After obtaining the fault information feedback matrix according to the above, the corresponding fault judgment matrix is obtained by multiplying the structure matrix and the fault information feedback matrix .When the column matrix is obtained, the local fault can be judged according to the element value of .The specific judgment rule is: when an element value of is equal to zero, according to Kirchhoff's current law, the current flowing into the area is equal to the outflow, and it can be judged that there is no fault; if an element value of is greater than zero, the current flowing into the area can be considered to be greater than the fault current, Can be judged as a fault area; if an element value of is less than zero, it is generally the area directly connected to the power supply, and it can be judged as a fault-free area.

That a ground fault occurs in area (1), according to the system power flow of the arrow shown in figure 2 , the fault feedback matrix can be. The fault judgment matrix is obtained by multiplying the matrix and the feedback matrix. According to the above fault judgment principle, the fault area is (1).

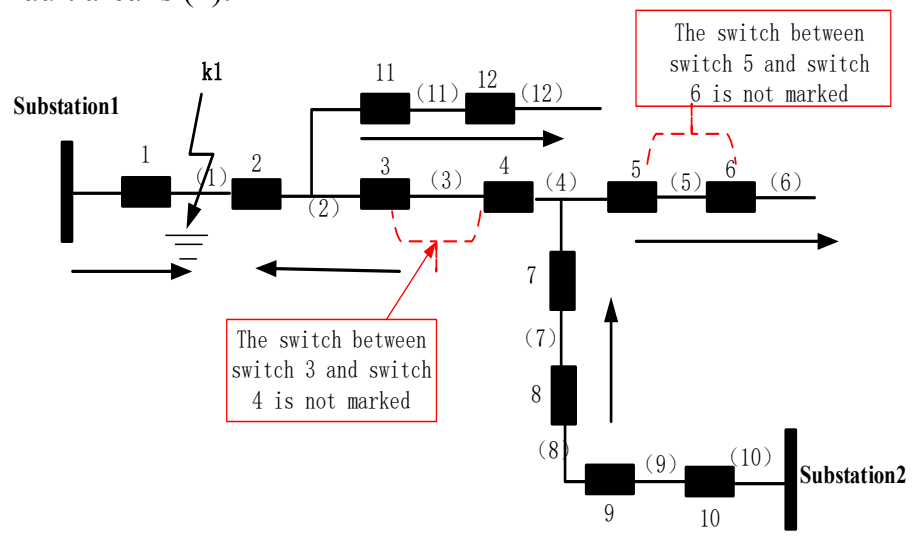

1-12:smart switch (1)-(12):line section

Figure 2. Distribution network area (1) fault k1

\subsection{Matrix algorithm considering error tolerance}

Due to the interference of the state of the transmission equipment and the external environment, the transmission of data will fail, and the accuracy of the data received by the monitoring terminal is low, which brings certain difficulties to the fault judgment. When a device fails to transmit data, the corresponding element of the fault feedback matrix is 0 . At this time, the structure matrix and the information feedback matrix need to be corrected to obtain a new fault judgment matrix. For example, if the local area (8) of the distribution network fails, as shown in figure 3, and the terminal equipment 3 fails to upload the fault information or the returned data cannot be updated, the fault range may be judged inaccurately or cannot be judged according to the data. The corresponding wrong data 
matrix shall be corrected, and the specific operation is as follows ${ }^{[1-12]}$.

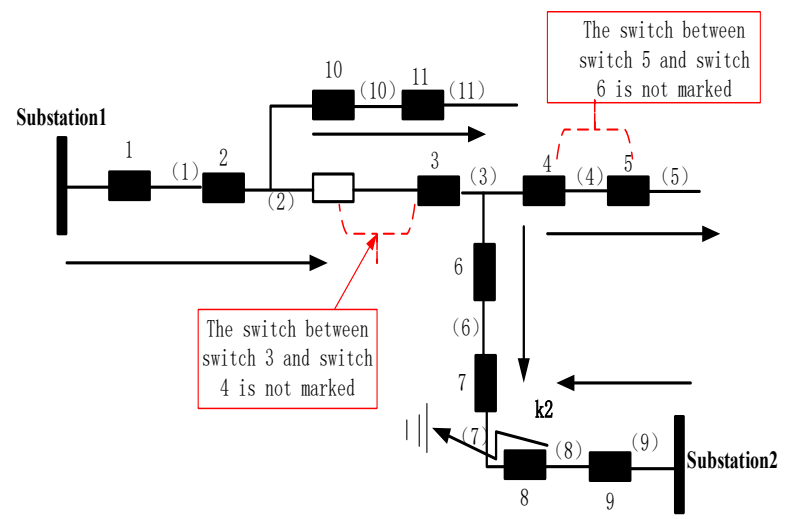

1-12:smart switch (1)-(12):line section

Figure 3. K2 fault and signal loss of switch 3 in distribution network

$$
Y_{30}=\left[\begin{array}{ccccccccccc}
1 & -1 & 0 & 0 & 0 & 0 & 0 & 0 & 0 & 0 & 0 \\
0 & 1 & -1 & 0 & 0 & 0 & 0 & 0 & 0 & -1 & 0 \\
0 & 0 & 1 & -1 & 0 & 1 & 0 & 0 & 0 & 0 & 0 \\
0 & 0 & 0 & 1 & -1 & 0 & 0 & 0 & 0 & 0 & 0 \\
0 & 0 & 0 & 0 & 1 & 0 & 0 & 0 & 0 & 0 & 0 \\
0 & 0 & 0 & 0 & 0 & -1 & 1 & 0 & 0 & 0 & 0 \\
0 & 0 & 0 & 0 & 0 & 0 & -1 & 1 & 0 & 0 & 0 \\
0 & 0 & 0 & 0 & 0 & 0 & 0 & -1 & 1 & 0 & 0 \\
0 & 0 & 0 & 0 & 0 & 0 & 0 & 0 & -1 & 0 & 0 \\
0 & 0 & 0 & 0 & 0 & 0 & 0 & 0 & 0 & 1 & -1 \\
0 & 0 & 0 & 0 & 0 & 0 & 0 & 0 & 0 & 0 & 1
\end{array}\right]
$$

Then a new structure matrix $Y_{30}$ is obtained by deleting the row and column of switch 3 corresponding to the structure matrix Y. The fault information matrix also eliminates the signal of switch 3 to generate a new fault information feedback matrix $D f_{30}=[1,1,1,1,1,-1,-1,1,1,1,1]^{T} ;$ According to the calculation of $G P=Y_{30} * D f_{30}$, the fault judgment matrix is $G P=[0,-1,0,0,1,0,2,0,-1,0,1]^{T}$. According to the value of the fault judgment matrix, the fault occurs in the (7) region. When the fault information is not perfect, it can correctly judge the fault area. However, this method is suitable for the case of multiple power supply, and is not feasible for single power supply. In the face of the actual operation of the distribution network, the single power supply is the majority, and the single power supply and dual power supply mode are often changed. Therefore, it is necessary to consider the use of dichotomy and improved matrix algorithm, make full use of the advantages of the two methods, can quickly find the distribution network line fault, strive for sufficient repair time for maintenance personnel, and reduce the scope of line blackout.

\section{Combination of dichotomy and improved matrix algorithm}

The dichotomy method is to establish the preset fault breaking point library of each line considering the number of line users. When the distribution network line breaks down, the 
dispatcher orders the distribution network emergency repair personnel to disconnect the preset isolation breaking point switch of the corresponding line according to the preset fault breaking point library, and the fault line is divided into two parts. Then, the power supply end of the line is tested. If the switch recloses and then trips, it indicates that the fault occurs in this section of the line. On the other hand, it is another line, after the fault line is determined, continue to according to the preset breaking point, and then use dichotomy to continue to narrow the fault range and quickly recover the non-fault line segment. As shown in figure 4, the distribution network lines, $A \backslash B$ are substations, $D$ is the switching station, the 1-4 number is the column switch, the $a / b$ is the substation outlet switch, $d 1 / d 2$ is the switch in the switching station, the switch on the pole is solid, the switch is in the closing position, and the hollow indicates that the switch is in the disconnected position ${ }^{[13]}$. When two faults are $\mathrm{k} 1$ and $\mathrm{k} 2$, the specific dichotomy operation steps are as follows:

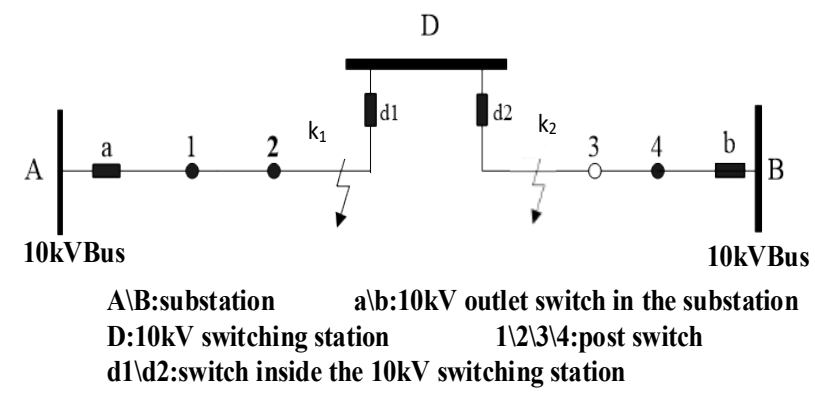

Figure 4. Schematic diagram of distribution network fault

Operation steps of dichotomy:

(1) Disconnect the d1 switch (the preset breaking point) of the $10 \mathrm{kV}$ switching station of the faulty line to segment the faulty line;

(2) Make the inspection team check that the substation switch is not abnormal, and try to send the segmented line;

(3) If the test transmission of the substation switch is unsuccessful, the test transmission will be conducted on the other side of the communication section.

(4) If the fault point is at the k1 point, the switch a fails to be tested, and the fault area is determined as the line between the switch a and the switch $\mathrm{d} 1$, then the power supply is resumed.

(5) If the fault point is at the $\mathrm{k} 2$ point, the a switch is successfully tested, the fault range is between the switch $\mathrm{d} 1$ and the switch 3 , and the users on the power side fault free circuit resume power transmission, and the subsequent line fault continues to be searched.

\section{Case analysis}

In order to verify the effectiveness of the proposed algorithm, this paper uses a multi-power mode distribution network in a certain city as shown in figure 5 to conduct simulation tests for single fault and multiple fault types of fault location.

At present, three different positions of distribution network are set with grounding fault, which are $\mathrm{k} 1, \mathrm{k} 2$ and $\mathrm{k} 3$. Through the fault test in three different places, the algorithm judges the fault area, and the test results are shown in Table 1. 


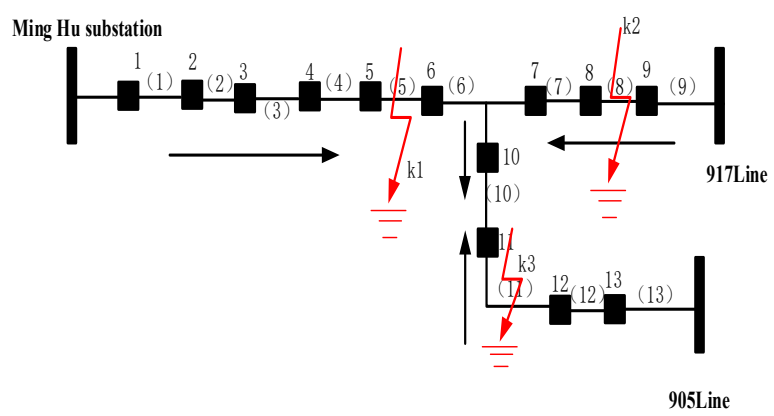

Figure 5. Multi-power operation mode of distribution network

Table 1. Example simulation results

\begin{tabular}{|c|c|c|c|c|}
\hline Fault information matrix & $\begin{array}{c}\text { Fault } \\
\text { type }\end{array}$ & Fault judgment matrix & $\begin{array}{c}\text { False negative } \\
\text { switch }\end{array}$ & $\begin{array}{c}\text { Fault } \\
\text { area }\end{array}$ \\
\hline$[1,1,1,1,1,-1,1,1,1,1,1,1,1]$ & $\mathrm{k} 1$ & {$[0,0,0,0,2,1,0,0,0,0,0,0,0]$} & naught & 5 \\
\hline$[1,1,1,1,1,1,-1,-1,1,1,1,1,1]$ & $\mathrm{k} 2$ & {$[0,0,0,0,0,1,0,2,0,0,0,0,0]$} & naught & 8 \\
\hline$[1,1,1,1,1,1,-1,-1,1,1,1,1,1]$ & $\mathrm{k} 3$ & {$[0,0,0,0,0,1,0,2,0,0,0,0,0]$} & naught & 11 \\
\hline$[1,1,1,1,1,-1,1,1,1,-1,-1,1,1]$ & $\mathrm{k} 1, \mathrm{k} 3$ & {$[0,0,0,0,2,-1,0,0,0,0,2,0,0]$} & naught & 5,11 \\
\hline$\left[\begin{array}{c}1,1,1,1,1,-1,1,1,1,-1,- \\
1,1,1]\end{array}\right.$ & $\mathrm{k} 2, \mathrm{k} 3$ & {$[0,0,0,0,2,-1,0,0,0,0,2,0,0]$} & naught & 8,11 \\
\hline$[1,1,1,0,1,-1,1,1,1,1,1,1,1]$ & $\mathrm{k} 1$ & {$[0,0,1,-1,2,1,0,0,0,0,0,0,0]$} & 4 & 5 \\
\hline$[1,1,1,0,1,-1,1,1,1,-1,-1,1,1]$ & $\mathrm{k} 1, \mathrm{k} 3$ & {$[0,0,1,-1,2,-1,0,0,0,0,2,0,0]$} & 4 & 5,11 \\
\hline$[1,1,1,0,1,-1,1,0,1,1,1,1,1]$ & $\mathrm{k} 1$ & {$[0,0,1,-1,2,1,-1,1,0,0,0,0,0]$} & 4,8 & 5 \\
\hline$[1,1,1,0,1,-1,1,0,1,-1,-1,1,1]$ & $\mathrm{k} 1, \mathrm{k} 3$ & {$[0,0,1,-1,2,-1,-1,1,0,0,2,0,0]$} & 4,8 & 5,11 \\
\hline
\end{tabular}

According to the results in Table 1, this method can effectively locate the fault area when single or multiple fault occurs in the distribution network of loop-circuit operation mode. Even when the feeder terminal equipment fails to report the signal, it can effectively locate the fault area. Finally, the specific location of the fault point is judged by dichotomy, and the power supply in the fault-free area is quickly restored. To sum up, from the occurrence of the fault to the restoration of all fault-free lines, this method not only reduces the number of insulation and switch operations of distribution emergency repair personnel, but also greatly reduces the operation risk caused by high-intensity work. At the same time, it reduces the number of dispatcher orders, reduces the possibility of wrong scheduling, and reduces the customer outage time.

\section{Conclusion}

According to the actual operation conditions of distribution network and the corresponding automation equipment, this paper puts forward an improved matrix bisection fault location method, which adapts to the distributed generation access to the distribution network, when its operation mode and network structure complexity increase, and make full use of the existing equipment to achieve accurate fault location. The analysis of the example shows that this method can also locate the fault in the case of automatic equipment fault tolerance and data transmission loss, which makes the distribution network operators quickly isolate 
the fault, expand and restore the power supply in the fault-free area, greatly reduce the fault location time, and improve the power supply reliability of the distribution network.

\section{References}

1. Wang Qiujie, Jin Tao, Liu Jun. Hierarchical analytic model for fault location of distribution network considering FTU missing and false positives [J]. Electric Power Automation Equipment, 2019, 39(01): 141-147.

2. Guo Yanyan, Xiong Guojiang. Fault section location in distribution network by means of sine cosine algorithm [J]. Power System Protection and Control, 2017, 45(13): 97101.

3. Hu Funian, Sun Shoujuan. Fault Location of Distribution Network by Applying Matrix Algorithm Based on Graph Theory [J]. Electric Power, 2016, 49(03): 94-98.

4. Wang Yifei, Jia Yanbing. Fault Location of Distribution Networks with DGs Based on an Improved Matrix Algorithm [J]. Computer Simulation, 2018, 35(04): 58-64.

5. Xu Biao, Yin Xianggen, Zhang Zhe, etc. Fault Location for Distribution Network Based on Matrix Algorithm and Optimization Algorithm[J]. Automation of Electric Power Systems, 2019, 43(05): 152-158.

6. Huang Jiale, Yang Guanlu. Modified matrix algorithm for fault section location of distribution network [J]. Power System Protection and Control, 2014, 42(11): 41-45.

7. Yang Lei, Chen Kai, Xiong Qi, etc. Distribution Network Fault Location Based on Information Distortion Correction[J]. Journal of Electric Power, 2019, 34(03): 229234 ..

8. Zhong Jianwei, Zhu Jianfeng, Huang Xiuchao, etc. Fault Location in Distribution Network Based on BBPSO Algorithm [J]. Proceedings of the CSU-EPSA, 2019, 31(03): 29-34.

9. Xu Yutao, Tan Zhukui, Lu Qiansu, etc. Application of Improved Bacteria Feeding Algorithm Based on Differential Evolution in Fault Location of Smart Distribution Network [J]. Power Systems And Big Data, 2018, 21(05):1-7 .

10. Zhang Dehua, Cai Jinding, Li Tianyou, etc. An Improved Incident Matrix Algorithm for Fault Locating in Distribution System[J]. Electric Power and Electrical Engineering, 2013, 33(04): 29-31.

11. Zhang Shengsi, Zhang Yuning, Chen Tangxian. Fault Location Optimization Algorithm Based on Distribution Automation Terminal[J]. Journal of China Three Gorges University, 2020, 42(04): 84-87.

12. Cheng Cuiying, Qu Yi, Zhu Jianliang, etc. Fault Location Algorithm Based on DIgSILENT Simulation with Distributed Generators [J]. Journal of Huaiyin Institute of Technology, 2020, 29(01): 32-37.

13. Ou Liquan, Lu Lianjie, Xie Yigong, etc. Application Study on Bisection Method of Rapid Restoration for Distribution Network Fault in Kunming Area [J]. Yunnan Electric Power, 2017, 45(03): 142-145. 